\title{
Additional role of second washing specimen obtained during single bronchoscopy session in diagnosis of pulmonary tuberculosis
}

Hongseok Yoo ${ }^{1 \dagger}$, Jae-Uk Song ${ }^{2 \dagger}$, Won-Jung Koh', Kyeongman Jeon', Sang-Won Um', Gee Young Suh', Man Pyo Chung ${ }^{1}$, Hojoong Kim', O Jung Kwon' ${ }^{1}$ Nam Yong Lee ${ }^{3}$, Sookyoung Woo ${ }^{4}$ and Hye Yun Park ${ }^{1 *}$

\begin{abstract}
Background: Flexible bronchoscopy with bronchial washing is a useful procedure for diagnosis of pulmonary tuberculosis (TB), when a patient cannot produce sputum spontaneously or when sputum smears are negative. However, the benefit of gaining serial bronchial washing specimens for diagnosis of TB has not yet been studied. Therefore, we conducted a retrospective study to determine the diagnostic utility of additional bronchial washing specimens for the diagnosis of pulmonary TB in suspected patients.
\end{abstract}

Methods: A retrospective analysis was performed on 174 patients [sputum smear-negative, $n=95$ (55\%); lack of sputum specimen, $n=79(45 \%)]$ who received flexible bronchoscopy with two bronchial washing specimens with microbiological confirmation of pulmonary TB in Samsung Medical Center, between January, 2010 and December, 2011.

Results: Pulmonary TB was diagnosed by first bronchial washing specimen in 141 patients (81\%) out of 174 enrolled patients, and an additional bronchial washing specimen established diagnosis exclusively in 22 (13\%) patients. Smear for acid-fast bacilli (AFB) was positive in 46 patients (26\%) for the first bronchial washing specimen. Thirteen patients (7\%) were positive only on smear of an additional bronchial washing specimen. Combined smear positivity of the first and second bronchial washing specimens was significantly higher compared to first bronchial washing specimen alone [Total cases: 59 (34\%) vs. 46 (26\%), p < 0.001; cases for smear negative sputum: 25 (26\%) vs. $18(19 \%), p=0.016$; cases for poor expectoration: 34 (43\%) vs. 28 (35\%), $p=0.031]$. The diagnostic yield determined by culture was also significantly higher in combination of the first and second bronchial washing specimens compared to the first bronchial washing. [Total cases: 163 (94\%) vs. 141 (81\%), p < 0.001; cases for smear negative sputum: 86 (91\%) vs. 73 (77\%), $p<0.001$; cases for poor expectoration: 77 (98\%) vs. 68 (86\%), $p=0.004]$.

Conclusions: Obtaining an additional bronchial washing specimen could be a beneficial and considerable option for diagnosis of TB in patients with smear-negative sputum or who cannot produce sputum samples.

Keywords: Bronchial washing, Bronchoscopy, Diagnosis, Tuberculosis

\footnotetext{
*Correspondence: kerrybe.park@samsung.com

${ }^{\dagger}$ Equal contributors

'Division of Pulmonary and Critical Care Medicine, Department of Medicine, Samsung Medical Center, Sungkyunkwan University School of Medicine, 81 Irwon-ro, Gangnam-gu, Seoul, Republic of Korea

Full list of author information is available at the end of the article
} 


\section{Background}

Pulmonary tuberculosis (TB) is a major public health challenge worldwide, with an estimated 8.8 million new cases and 1.45 million TB-related deaths in 2010 [1]. Although various diagnostic tests for pulmonary TB have been developed, its definitive diagnosis still relies on culturing Mycobacterium tuberculosis (M. tuberculosis) from respiratory secretions. For sputum samples, international guidelines recommend obtaining at least two specimens from patients with suspected tuberculosis to increase the diagnostic sensitivity for identification of $M$. tuberculosis [2]. In patients with negative sputum smear or who cannot produce sputum samples, previous studies have proved efficacy of an alternative method of obtaining respiratory samples by bronchoscopy in diagnosis of TB [3-8]. However, it is yet known whether an additional bronchial specimen acquired from bronchial washing has an incremental diagnostic yield for diagnosis of TB as second and third sputum samples have [9-11]. Therefore, the aim of this study was to assess the diagnostic value of the second bronchial washing sequentially obtained after the first bronchial washing specimen in suspected pulmonary TB patients who are unable to expectorate or have pre-bronchoscopy smearnegative sputum.

\section{Methods}

\section{Study population}

This was a retrospective observational study on the role of additional bronchial washing specimens in diagnosis of TB in Samsung Medical Center (a 1960-bed, university-affiliated, tertiary referral hospital in Seoul, South Korea) between January 2010 and December 2011. TB case is clinically suspected as a patient currently not receiving tuberculosis treatment with a persistent cough for $>3$ weeks or symptoms consistent with TB (chest pain, low-grade fever, night sweats, shortness of breath, and weight loss) with chest radiograph or computed tomograph scan suggestive of pulmonary TB. In clinically suspected TB patients with smear-negative sputum or who are difficult to produce sputum samples, we obtain three serial bronchial washing samples during bronchoscopy.

Of those who underwent bronchoscopy with two bronchial washing specimens for AFB smear and culture, we recruited 225 patients with a microbiological confirmation of $M$. tuberculosis during the study period. Of those patients, 51 receiving tuberculosis treatment before bronchoscopy were excluded from the study. This left a population of 174 patients with a diagnosis of pulmonary tuberculosis from bronchial washing $(n=163)$; other methods, such as pathologic confirmation with culture from tissue obtained by bronchoscopy, $(\mathrm{n}=4)$; nucleic acid amplification assay for $M$. tuberculosis on the third bronchial washing $(\mathrm{n}=2)$; and sputum culture $(\mathrm{n}=5)$ (see Figure 1). Demographic, clinical and radiological variables were determined by retrospective analysis of medical records. The Institutional Review Board of Samsung Medical Center approved the review and publication of data obtained from the patients' records (Protocol No: 2011-07-037) and the study was conducted in compliance with the Helsinki Declaration. Informed consent was waived due to the retrospective nature of this study.

\section{Bronchoscopy procedures}

Bronchoscopy with bronchial washing was performed according to the standard technique as described previously $[12,13]$. Patients were sedated with intravenous midazolam. Under conscious sedation, the bronchoscope (Olympus, Tokyo, Japan) was inserted trans-nasally or orally and lidocaine of $5 \mathrm{~mL}$ to $10 \mathrm{~mL}$ as needed was sprayed intratracheally. After placing bronchoscope at the targeted segment, bronchial washing was performed by instilling $10 \mathrm{~mL}$ of warm sterile saline. Several breaths were allowed for the fluid to pool at the target segment before retrieval. This procedure was repeated twice at the same segment. Each retrieved sample was labeled as the first, second, and third sample. First and second samples were then tested for acid-fast bacilli (AFB) by smear and culture. The third specimen was subjected to a nucleic acid amplification assay for $M$. tuberculosis $[14,15]$. When multiple pulmonary infiltrates were visible on chest radiography, bronchial washing was performed at the segment exhibiting the most severe abnormalities.

\section{Microbiological examination}

Aliquots of specimens were decontaminated with the $2 \% \mathrm{~N}$-acetyl-L-cysteinhydroxide (NALC-NaOH). Then the specimens were stained by auramine-rhodamine fluorescent and Ziehl-Neelsen stain and examined under microscopy. The processed specimens were also plated onto both 3\% Ogawa solid medium (Shinyang, Seoul, Korea) and liquid culture medium (MGIT 960 system, Becton Dickinson, Sparks, MD, USA) and were incubated at $36^{\circ} \mathrm{C}$ for 6 weeks. Nucleic acid amplification test for M. tuberculosis was performed using COBAS TaqMan MTB assay (Roche Diagnostics, Basel, Switzerland) according to the manufacturer's guideline.

\section{Definition}

Microbiological confirmation of M. tuberculosis was defined as growth of $M$. tuberculosis or positive test result of the nucleic acid amplification for $M$. tuberculosis from respiratory or tissue samples. Immunocompromised condition was defined as following: undergoing chemotherapy for an malignancy within 4 weeks, being 


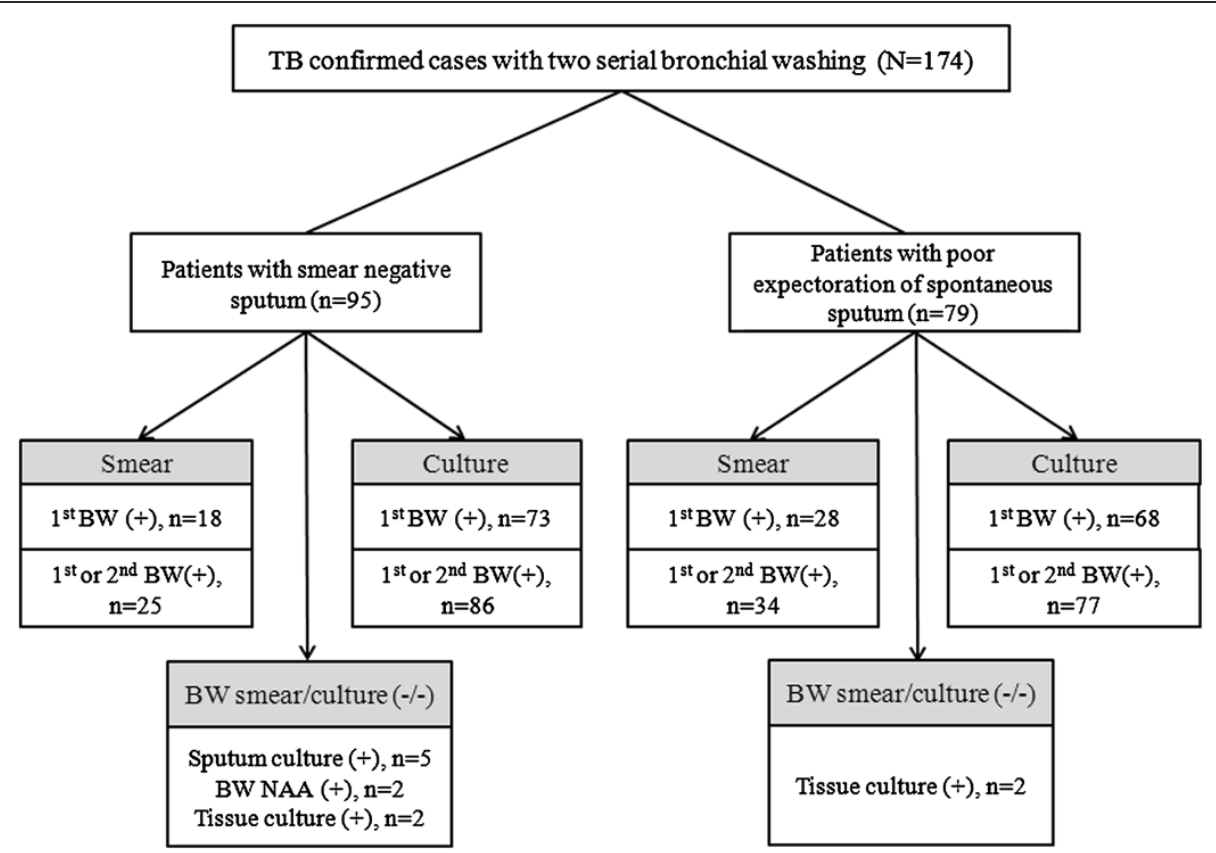

Figure 1 Diagnostic flow of 174 study patients. BW, bronchial washing; NAA = nucleic acid amplification.

seropositive for human immunodeficiency virus or undergoing daily administration of systemic corticosteroids (at least $20 \mathrm{mg}$ prednisolone or equivalent for more than 1 month), combination therapy with low dose corticosteroids and other immunosuppressants including azathioprine, mycophenolate, methotrexate, cyclosporine, or cyclophosphamide or under status of solid or hematologic transplantation.

\section{Statistical analysis}

All data are presented as numbers (percentages) for categorical variables and as medians with interquartile ranges (IQR) for continuous variables. The diagnostic yield of repeated bronchial washing for the diagnosis of TB was determined. The diagnostic consistency according to the number of bronchial washing was analyzed using the McNemar's test. Diagnostic yield and diagnostic consistency were also sub-analyzed based on the reason of bronchoscopy. To identify the factors to predict diagnostic yield according to the number or order of bronchial washing specimens, clinical and laboratory characteristics according to positivity of first and/or second bronchial washing specimens were compared using the Kruskal-Wallis test for continuous variables and the $\chi^{2}$ test for categorical variables. Statistical analyses were performed using IBM SPSS Statistics 21.0 (IBM, Chicago, IL, USA), and two-sided $P<0.05$ was considered significant.

The incremental yield of an additional bronchial washing specimen was defined as [(number of cases additionally diagnosed by second bronchial washing specimen/number of all diagnosed cases) $\times 100]$ [16]

\section{Results}

The baseline characteristics of the patients are summarized in Table 1. There were 90 males and 84 females, with a median age of 53 (IQR 34-68) years. Seventy six (44\%) patients had one or more comorbidities and 32 (18\%) had undergone previous TB treatment. The most common presenting symptoms were cough $(\mathrm{n}=43,25 \%)$ and sputum ( $n=23,13 \%)$. Chest radiographs were available for analysis in all patients. The most common radiologic finding was small nodules $(\mathrm{n}=95,55 \%)$, followed by consolidation $(\mathrm{n}=45,26 \%)$, atelectasis $(\mathrm{n}=19,11 \%)$, and cavitation ( $\mathrm{n}=18,10 \%$ ). The most common sites at which bronchial washing was performed were the right upper lobe $(n=71$, $41 \%)$ and the left upper lobe $(\mathrm{n}=49,28 \%)$. Regarding adverse events, five patients (2.9\%) had fever after bronchoscopy and one $(0.6 \%)$ had tachycardia, which resolved after bronchoscopy.

Out of 174 study patients, 46 (26\%) patients had a positive AFB smear in the first bronchial washing specimen, and additional 13 (7\%) patients had a positive AFB smear only in the second bronchial washing specimen. Nucleic acid amplification assay for M. tuberculosis using the third bronchial washing was positive in 72 (41\%) patients and was the sole method to diagnose pulmonary TB in 2 patients. Combined smear positivity of the first and second bronchial washing specimens was significantly higher than that of the first bronchial 
Table 1 Baseline characteristics of 174 study patients

\begin{tabular}{|c|c|}
\hline & No. of patients (\%) or median (IQR) \\
\hline Age, years & $53(34-68)$ \\
\hline Gender, female & $84(48)$ \\
\hline Comorbidity & $76(44)$ \\
\hline Malignancy & $31(18)$ \\
\hline Cardiovascular disease & $29(17)$ \\
\hline DM & $25(14)$ \\
\hline Chronic hepatitis & $3(2)$ \\
\hline Respiratory disease & $4(2)$ \\
\hline Rheumatic disease & $3(2)$ \\
\hline Transplantation & $2(1)$ \\
\hline Others* & $6(3)$ \\
\hline \multicolumn{2}{|l|}{ Smoking } \\
\hline Ex-smoker & $26(15)$ \\
\hline Current smoker & $41(24)$ \\
\hline Previous history of TB treatment & $32(18)$ \\
\hline Symptoms & $75(43)$ \\
\hline Cough & $43(25)$ \\
\hline Sputum & $23(13)$ \\
\hline Fever & $15(9)$ \\
\hline Hemoptysis & $11(6)$ \\
\hline Chest pain & $9(5)$ \\
\hline Dyspnea & $7(4)$ \\
\hline Sweating & $2(1)$ \\
\hline \multicolumn{2}{|l|}{ Chest radiology } \\
\hline Small nodules & $95(55)$ \\
\hline Consolidation & $45(26)$ \\
\hline Atelectasis & $19(11)$ \\
\hline Cavitation & $18(10)$ \\
\hline Mass & $14(8)$ \\
\hline Effusion & $9(5)$ \\
\hline Fibrotic scar & $8(5)$ \\
\hline Normal & $3(2)$ \\
\hline \multicolumn{2}{|l|}{ Location of bronchial washing } \\
\hline Right upper lobe & $71(41)$ \\
\hline Right middle lobe & $19(11)$ \\
\hline Right lower lobe & $25(14)$ \\
\hline Left upper lobe & $49(28)$ \\
\hline Left lower lobe & $10(6)$ \\
\hline
\end{tabular}

Abbreviations: $I Q R$, interquartile ranges; $D M$, diabetes mellitus; $T B$, tuberculosis. * Hyperthyroidism $(n=2)$, hypothyroidism $(n=1)$, paraplegia due to spinal cord injury $(n=1)$, Klippel-Feli syndrome $(n=1)$, osteoporosis $(n=1)$. Categorical variables were denoted by No. of patients (\%). Continuous variable was denoted by median (IQR). washing specimen alone [59 (34\%) vs. $46(26 \%), \mathrm{p}<$ 0.001]. (Figure 2) On culture, M. tuberculosis was identified in 141 (81\%) patients by first bronchial washing specimen. Additional bronchial washing specimen identified M. tuberculosis exclusively in $22(13 \%)$ patients. The diagnostic yield of culture was also significantly higher in combination of the first and second bronchial washing specimens compared to the first bronchial washing [163 (94\%) vs. 141 (81\%), p < 0.001]. (Figure 3).

Diagnostic yield was assessed based on the reason of bronchoscopy. Out of 95 patients with smear-negative sputum, 18 patients (19\%) had a positive AFB smear in the first bronchial washing and an additional seven (7\%) had a positive AFB smear from the second bronchial washing. Combined smear positivity of the first and second bronchial washing specimens was higher than that of first bronchial washing specimen alone [25 (26\%) vs. $18(19 \%), \mathrm{p}=0.016]$. (Figure 2) On culture, M. tuberculosis was identified in 73 patients from the first bronchial washing and 86 patients had pulmonary TB on culture from combination of the first and second bronchial washing [86 (91\%) vs. 73 (77\%), $p<0.001$ ]. (Figure 3) The incremental yield of culture positive cases was $14 \%$ with the additional second bronchial washing. Of 79 patients who were unable to expectorate, an AFB smear by direct microscopy was positive in 28 patients (35\%) for the first bronchial washing and six (8\%) were positive upon an additional bronchial washing smear. Cultures positive for $M$. tuberculosis were obtained in 68 patients (86\%) using first bronchial washing specimens, and the first or second bronchial washing specimen was diagnostic in 77 patients (98\%). Accordingly, the incremental yield from the second bronchial washing was $11 \%$. The diagnostic yield for smear and culture of the combined bronchial washing specimens were significantly higher compared to the first bronchial washing specimen [smear: $34(43 \%)$ vs. 28 (35\%), p = 0.031; culture: 77 (98\%) vs. 68 (86\%), p < 0.001]. (Figure 2 and 3).

A drug susceptibility test was performed in 172 patients, excluding two who were diagnosed by a positive nucleic acid amplification test without culture of $M$. tuberculosis. Of 141 patients first bronchial washing of whom was cultured, 20 had drug-resistant tuberculosis (13 had multi-drug resistance (MDR) and seven had isoniazid (INH) resistance (INH-R)). The second bronchial washing identified seven additional patients with drugresistant tuberculosis (four with MDR including one with extensive drug-resistance (XDR) and three with INH-R).

Table 2 shows comparisons of the clinical and radiological characteristics of patient groups with a positive result only on the first bronchial washing, only on the second bronchial washing, and in both bronchial washings $(n=163)$. There were no significant factors to 


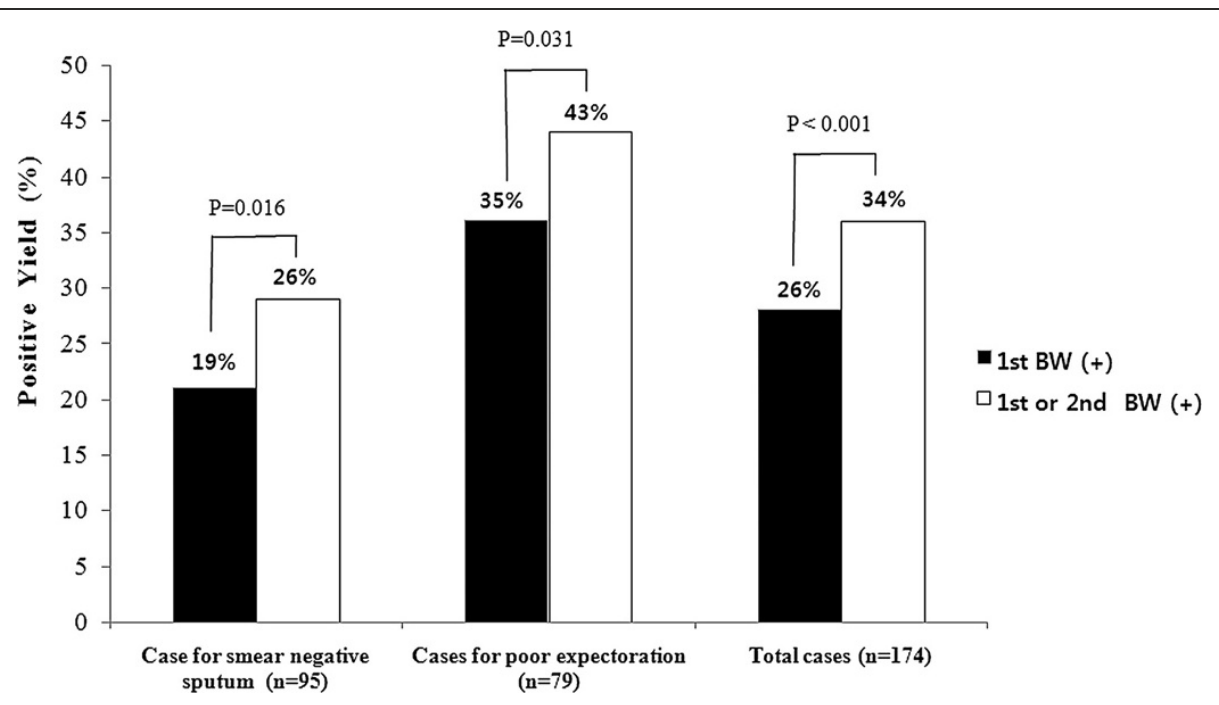

Figure 2 Smear positive yield of single bronchial washing and two consecutive bronchial washing specimens. BW, bronchial washing.

predict diagnostic yield according to the number or order of bronchial washing specimens, including age, gender, comorbidities, smoking history, previous TB history or immune status. There were also no significant differences among groups regarding radiographic findings, as well.

\section{Disscusion}

To our knowledge, this is the first study of the diagnostic value of an additional bronchial specimen acquired after the first bronchial washing. This retrospective study demonstrated that the diagnosis of pulmonary tuberculosis was increased by up to $13 \%$ with second bronchial washings in subjects whose spontaneous sputum was smear negative or who could not produce spontaneous sputum. In addition, second bronchial washing specimen identified additional drug-resistant $M$. tuberculosis in 7 patients (four MDR pathogens including one XDR and three INH-R pathogens).

It is well recognized that the smear positivity yield in sputum increases as the number of sputum samples increases in patients suspected as having pulmonary TB. A large study conducted in Tanzania showed that an incremental yield of $83.4 \%$ with the first, $12.2 \%$ with the second, and $4.4 \%$ with the third smear was estimated for the total number of expected cases (combined suspects with a complete set of three sputum smears examined and one with an incomplete examination) [11]. In Malawi, all TB suspects submit three sputum specimens for smear examination and the diagnosis is made by the first specimen in $83 \%$, the second specimen in $13 \%$, and the third specimen in $4 \%$ of patients [17]. Given the high

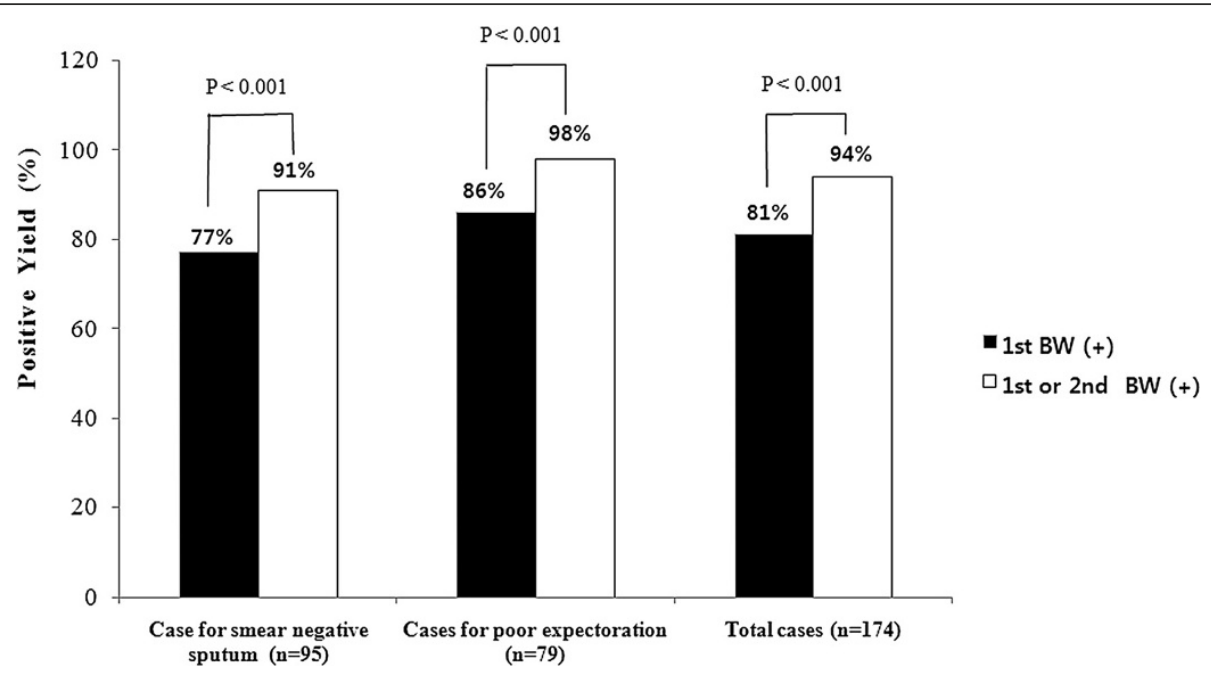

Figure 3 Culture positive yield of single bronchial washing and two consecutive bronchial washing specimens. BW, bronchial washing. 
Table 2 Comparisons of the clinical and radiological characteristics according to positive result in serial bronchial washing specimens in 163 patients*

\begin{tabular}{|c|c|c|c|c|}
\hline & BW1 (+) $(n=30)$ & BW2 $(+)(n=22)$ & BW1 \& $2(+)(n=111)$ & $P$ value \\
\hline Age, years & $54(34-71)$ & $60(29-71)$ & $52(34-68)$ & 0.898 \\
\hline Gender, female & $11(37)$ & $12(54)$ & $57(51)$ & 0.310 \\
\hline Presence of Comorbidity & $11(37)$ & $10(46)$ & $51(46)$ & 0.657 \\
\hline Immunocompromised patients & $1(3)$ & $1(5)$ & $11(10)$ & 0.407 \\
\hline Current of ex-smoker & $12(40)$ & $9(41)$ & $42(38)$ & 0.951 \\
\hline Previous history of treatment TB & $5(17)$ & $4(18)$ & $23(21)$ & 0.869 \\
\hline Clinical Symptom & $13(43)$ & $8(36)$ & $51(46)$ & 0.707 \\
\hline No symptoms & $17(57)$ & $14(64)$ & $60(54)$ & 0.707 \\
\hline \multicolumn{5}{|l|}{ Chest radiology } \\
\hline Fibrotic scar & $1(3)$ & 0 & $6(5)$ & 0.500 \\
\hline Cavitation & $3(10)$ & $5(23)$ & $10(9)$ & 0.169 \\
\hline Nodules & $16(53)$ & $17(77)$ & $58(52)$ & 0.093 \\
\hline Mass & $5(17)$ & 0 & $7(6)$ & 0.057 \\
\hline Consolidation & $6(20)$ & $3(14)$ & $34(31)$ & 0.174 \\
\hline Atelectasis & $1(3)$ & $3(14)$ & $13(12)$ & 0.358 \\
\hline Effusion & $1(3)$ & $1(5)$ & $6(5)$ & 0.894 \\
\hline Drug resistance & $3(10)$ & $7(32)$ & $17(15)$ & 0.092 \\
\hline
\end{tabular}

$B W$, Bronchial washing.

Categorical variables were denoted by No. of patients (\%).

Continuous variable was denoted by median (IQR).

*Patients with positive results for $M$. tuberculosis from bronchial washing.

diagnostic yield of over $10 \%$ in the second sputum smear [18] and the cost-effectiveness of obtaining the third specimen in low-income countries, [19] international guidelines recommend to obtain at least two sputum specimens from patients with suspected tuberculosis to increase the diagnostic yield for identification of $M$. tuberculosis [2]. In line with previous studies that used serial sputum samples, $[11,17,18]$ our findings demonstrated for the first time a 13\% culture incremental yield from an additional bronchial washing compared with performing only a single bronchial washing in patients with smear-negative sputum or in those in whom sputum was unavailable. Although no other reports or universal cut-off values are available for comparison with our results, we believe that an incremental culture rate of $13 \%$ for the second bronchial washing highlights the importance of additional bronchial washings.

In our study, consecutive first and second bronchial washings provided high TB diagnostic yield in patients who were negative on sputum smear microscopy or were unable to expectorate sputum; culture for $M$. tuberculosis was positive in 163 of 174 patients (94\%). When our patients were divided according to the reason for performance of bronchoscopy, $77 \%$ of those who were negative on sputum smear microscopy were culture-positive in the first bronchial washing, which is consistent with previous reports of a positivity rate of $65-78 \%$ for culture of a single bronchial washing $[7,8]$. Use of a second bronchial washing added $8 \%$ to the positivity rate of culture for diagnosis of pulmonary $\mathrm{TB}$, leading to a diagnostic yield of $85 \%$ of suspected TB patients with smear-negative sputum. In patients who were unable to expectorate sputum, however, few studies have focused on the diagnostic power of bronchial washing, while our data showed that consecutive first and second bronchial washings led to a rapid diagnosis in $43 \%$ by microscopy smear and accounted for the culture positivity of 77 of 79 patients (98\%). Thus, these data indicate the diagnostic importance of bronchial washing in patients who lack spontaneous sputum for TB diagnosis.

In our study, the yield of $M$. tuberculosis culture was almost three times that of AFB smears, confirming the importance of culture for the diagnosis of pulmonary TB in our patients. In an era of increasing drug resistance, isolation of $M$. tuberculosis from respiratory specimens is important for drug sensitivity test. Additional 7 (26\% of all drug- resistant TB) patients have been confirmed to have drug-resistant tuberculosis in the present study by means of further secondary bronchial washing. It indicates that an additional bronchial washing is a meaningful process not only in increasing the diagnostic yield but also in obtaining $M$. tuberculosis strain which allows drug susceptibility test.

For patients with positive results for $M$. tuberculosis from bronchial washing, there are no specific clinical or 
radiologic parameters which can determine who needs second bronchial washing. The reported adverse events associated with the procedure were tolerable. Furthermore, the inhibitory effect of lidocaine on the growth of $M$. tuberculosis might contribute to the false negative rate and/or relatively low colony counts of $M$. tuberculosis from first bronchial washing specimens [20]. Thus, we suggest that obtaining two serial bronchial samples is safe and reasonable during performance of bronchoscopy in patients with smear-negative sputum or in those who cannot produce sputum samples.

To fully appreciate these results, the limitations of this study must be acknowledged. First, given the retrospective design of this study, it is possible that selection bias influenced our findings. A prospective study would be needed to fully validate these promising findings. Additionally, the increased yield of TB diagnosis in our study might have been related to larger cumulative volume rather than the repeated washing process. A prospective study comparing separate bronchial washings and single bronchial washing with the same amount of total saline is required to address this question. Third, our study was conducted at a single institution in an area of intermediate TB burden (incidence of TB, 97 per 100,000), which might limit the application of our findings, especially in areas of differing TB prevalence. Finally, we were unable to evaluate the economic cost associated with the study protocol or the counterproductive effects of overburdened technicians. High costs and technician shortages may limit the use of bronchoscopy in other situations.

\section{Conclusions}

In summary, we found that obtaining additional second bronchial washing sample increased the diagnostic yield by up to $13 \%$ under tolerable adverse events. This suggests that two serial bronchial washings are a beneficial and reasonable option for detection of TB and evaluation of drug resistance in patients who have negative AFB smears or who are unable to produce sputum samples in intermediate TB-burden countries.

\footnotetext{
Abbreviations

AFB: Acid-fast bacilli; M. tuberculosis: Mycobacterium tuberculosis; TB: Tuberculosis; MDR: Multi-drug resistance; INH: Isoniazid; INH-R: Isoniazid resistance; XDR: Extensive drug-resistance.
}

\section{Competing interests}

The authors declare that they have no competing interests.

\section{Authors' contributions}

HYP and WJK conceived the initial idea and the study design; JUS, HY, SWU, KJ, SW and HYP linked the data, contributed to data analysis and results interpretation; JUS and HY draft the manuscript; GYS, MPC, HK, OJK, NYL and HYP revised manuscript. All authors read and approved the final manuscript.

\section{Acknowledgements}

The authors would like to thank nurses Jung Eun Park and Soo Jin Park of bronchoscopy room for their assistance in performing the bronchoscopy. The authors would like to express their gratitude to Mr. Sung Eun James Lee and Ms. Wonyoung Yu for their aid in editing and proofreading this manuscript.

\section{Author details}

${ }^{1}$ Division of Pulmonary and Critical Care Medicine, Department of Medicine, Samsung Medical Center, Sungkyunkwan University School of Medicine, 81 Irwon-ro, Gangnam-gu, Seoul, Republic of Korea. ${ }^{2}$ Division of Pulmonary and Critical Care Medicine, Department of Medicine, Kangbuk Samsung Hospital, Sungkyunkwan University School of Medicine, Seoul, Republic of Korea.

${ }^{3}$ Department of Laboratory Medicine and Genetics, Samsung Medical Center, Sungkyunkwan University School of Medicine, Seoul, Republic of Korea.

${ }^{4}$ Biostatistics Team, Samsung Biomedical Research Institute, Seoul, Republic of Korea.

Received: 1 May 2013 Accepted: 26 August 2013

Published: 2 September 2013

\section{References}

1. Global Tuberculosis Control 2011. Geneva: WHO; 2011. http://www.who.int/ tb/publications/global_report/2011/gtbr11_full.pdf [last accessed 2012 Jul 24.

2. WHO: Treatment of Tuberculosis: guidelines. 4th edition. WHO; 2010. http://whqlibdoc.who.int/publications/2010/9789241547833_eng.pdf.

3. Willcox PA, Benatar SR, Potgieter PD: Use of the flexible fibreoptic bronchoscope in diagnosis of sputum-negative pulmonary tuberculosis. Thorax 1982, 37(8):598-601.

4. Wongthim S, Udompanich V, Limthongkul S, Charoenlap P, Nuchprayoon C: Fiberoptic bronchoscopy in diagnosis of patients with suspected active pulmonary tuberculosis. J Med Assoc Thai 1989, 72(3):154-159.

5. Charoenratanakul S, Dejsomritrutai W, Chaiprasert A: Diagnostic role of fiberoptic bronchoscopy in suspected smear negative pulmonary tuberculosis. Respir Med 1995, 89(9):621-623.

6. Mohan A, Sharma SK: Fibreoptic bronchoscopy in the diagnosis of sputum smear-negative pulmonary tuberculosis: current status. Indian J Chest Dis Allied Sci 2008, 50(1):67-78.

7. Altaf Bachh A, Gupta R, Haq I, Varudkar HG: Diagnosing sputum/smearnegative pulmonary tuberculosis: Does fibre-optic bronchoscopy play a significant role? Lung India 2010, 27(2):58-62.

8. Shin JA, Chang YS, Kim TH, Kim HJ, Ahn CM, Byun MK: Fiberoptic bronchoscopy for the rapid diagnosis of smear-negative pulmonary tuberculosis. BMC Infect Dis 2012, 12(1):141.

9. Enarson DA, Rieder HL, Arnadottir A, Trébucq A: Management of tuberculosis. A guide for low income countries. 5th edition. Paris: International Union Against Tuberculosis and Lung Disease; 2000.

10. Rieder HL, Chiang CY, Rusen ID: A method to determine the utility of the third diagnostic and the second follow-up sputum smear examinations to diagnose tuberculosis cases and failures. Int J Tuberc Lung Dis 2005, 9(4):384-391.

11. Ipuge YA, Rieder HL, Enarson DA: The yield of acid-fast bacilli from serial smears in routine microscopy laboratories in rural Tanzania. Trans $R$ Soc Trop Med Hyg 1996, 90(3):258-261.

12. Feinsilver SF: AM: Textbook of Bronchoscopy. 1st edition. Baltimore: Lippincott Williams \& Wilkins; 1995.

13. Kim MH, Suh GY, Chung MP, Kim H, Kwon OJ, Lee JH, Lee NY, Koh WJ: The value of routinely culturing for tuberculosis during bronchoscopies in an intermediate tuberculosis-burden country. Yonsei Med J 2007, 48(6):969-972.

14. Al Zahrani K, Al Jahdali H, Poirier L, Rene P, Menzies D: Yield of smear, culture and amplification tests from repeated sputum induction for the diagnosis of pulmonary tuberculosis. Int J Tuberc Lung Dis 2001, 5(9):855-860.

15. Laraque F, Griggs A, Slopen M, Munsiff SS: Performance of nucleic acid amplification tests for diagnosis of tuberculosis in a large urban setting. Clinical infectious diseases: an official publication of the Infectious Diseases Society of America 2009, 49(1):46-54.

16. Nataraj $G$, Kanade S, Parikh R, Khatri V, Mehta P, Athavale A, Arun B: Incremental yield in sputum smear positivity by examining a second 
early morning sputum specimen in follow-up patients on DOTS: 7 year analysis of RNTCP laboratory register. Indian J Tuberc 2011, 58(2):60-65.

17. Harries AD, Kamenya A, Subramanyam VR, Salaniponi FM, Nyangulu DS: Sputum smears for diagnosis of smear-positive pulmonary tuberculosis. Lancet 1996, 347(9004):834-835.

18. Wu ZL, Wang AQ: Diagnostic yield of repeated smear microscopy examinations among patients suspected of pulmonary TB in Shandong province of China. Int J Tuberc Lung Dis 2000, 4(11):1086-1087.

19. Harries AD, Mphasa NB, Mundy C, Banerjee A, Kwanjana JH, Salaniponi FM: Screening tuberculosis suspects using two sputum smears. Int I Tuberc Lung Dis 2000, 4(1):36-40.

20. Schmidt RM, Rosenkranz HS: Antimicrobial activity of local anesthetics: lidocaine and procaine. J Infect Dis 1970, 121(6):597-607.

\section{Submit your next manuscript to BioMed Central} and take full advantage of:

- Convenient online submission

- Thorough peer review

- No space constraints or color figure charges

- Immediate publication on acceptance

- Inclusion in PubMed, CAS, Scopus and Google Scholar

- Research which is freely available for redistribution 